\author{
Shunji Tomatsu · Tatiana Dieter · Ida V. Schwartz \\ Piedad Sarmient · Roberto Giugliani · Luis A. Barrera \\ Norberto Guelbert · Raquel Kremer \\ Gabriela M. Repetto - Monica A. Gutierrez \\ Tatsuo Nishioka $\cdot$ Olga Peña Serrato \\ Adriana Maria Montaño $\cdot$ Seiji Yamaguchi \\ Akihiko Noguchi

\section{Identification of a common mutation in mucopolysaccharidosis IVA: correlation among genotype, phenotype, and keratan sulfate}

Received: 28 April 2004/ Accepted: 7 June 2004/Published online: 11 August 2004

(C) The Japan Society of Human Genetics and Springer-Verlag 2004

\begin{abstract}
Mucopolysaccharidosis IVA (MPS IVA) is a lysosomal storage disorder caused by the deficiency of $N$-acetylgalactosamine-6-sulfate sulfatase (GALNS). Mutation screening of the GALNS was performed by genomic PCR and direct sequence analyses in 20 MPS IVA patients from Latin America. In this study, 12 different gene mutations including nine unreported ones were identified in 16 severe and four attenuated patients and accounted for $90.0 \%$ of the unrelated mutant alleles. The gene alterations were missense mutations except one insertion. Six recurrent mutations, p.A75G, p.G116S, p.G139S, p.N164T, p.R380S,
\end{abstract}

S. Tomatsu $(\bowtie) \cdot$ M. A. Gutierrez · T. Nishioka · O. P. Serrato · A. Noguchi

Department of Pediatrics, Pediatric Research Institute,

Saint Louis University, 3662 Park Ave., St Louis, MO, 63110-2586, USA

E-mail: tomatsus@slu.edu

T. Dieter $\cdot$ I. V. Schwartz $\cdot$ R. Giugliani

Medical Genetics Service, Hospital de Clinicas de Porto Alegre, Porto Alegre, RS, Brazil

P. Sarmient · L. A. Barrera · M. A. Gutierrez Institute of Inborn Errors of Metabolism, Javeriana University, Bogota, Colombia

N. Guelbert · R. Kremer

CEMECO, Centro de Estudio de las Metabolopatias Congenitas, Hospital de Ninos de Cordoba, Cordoba, Argentina

G. M. Repetto

Facultad de Ciencias de la Salud,

Universidad del Desarrollo-Clinica Alemana,

Santiago, Chile

\section{A. M. Montaño}

Department of Biosystems Science,

The Graduate University for Advanced Studies,

Kanagawa, Japan

S. Yamaguchi

Department of Pediatrics, Shimane University,

Izumo, Japan and p.R386C, accounted for 5.0, 10.0, 5.0, 7.5, 5.0, and $32.5 \%$ of the unrelated mutant alleles, respectively. The p.R386C mutation was identified in all Latin American populations studied. Eleven mutations correlated with a severe form, while one mutation, p.R380S, was associated with an attenuated form. MPS IVA patients had an elevation of urine and plasma keratan sulfate (KS) concentrations compared with those of the agematched control. KS concentrations in severe patients were higher than those in attenuated patients. These data provide evidence for extensive allelic heterogeneity and presence of a common mutation in Latin American patients. Accumulation of mutations with clinical description and KS concentration will lead us to predict clinical severity of the patient more precisely.

Keywords $N$-acetylgalactosamine-6-sulfate sulfatase * Mucopolysaccharidosis IVA · Common mutation · Genomic PCR amplification · Latin America

\section{Introduction}

Mucopolysaccharidosis type IVA (MPS IVA: Morquio type A) is an autosomal recessive disorder caused by the deficiency of lysosomal $\mathrm{N}$-acetylgalactosamine-6-sulfate sulfatase (GALNS: E.C.3.1.6.4; MIM\# 253000). GALNS is one of several sulfatases required to degrade glycosaminoglycans (GAGs), keratan sulfate (KS), and chondroitin-6-sulfate (C6S). Phenotypes of MPS IVA patients vary from the classical form with severe bone dysplasia, short trunk dwarfism, hearing loss, heart valve involvement, corneal opacity, and a life span of 20-30 years, to the attenuated form with fewer manifestations. The GALNS cDNA is 2,339 bp in length with a 1,566 bp open-reading frame encoding 522 amino 
acids (Tomatsu et al. 1991), and the gene for GALNS is split into 14 exons spanning approximately $50 \mathrm{~kb}$ (Nakashima et al. 1994) (GenBank: NM_000512.2, NT_010542.14).

Molecular analyses of MPS IVA patients were carried out in diverse ethnic origins, especially European and Japanese ancestries (Fukuda et al. 1992; Tomatsu et al. 1995; Ogawa et al. 1995; Bunge et al. 1997; Yamada et al. 1998; Montano et al. 2003). However, little has been reported on MPS IVA patients in the Latin American population. The principal aim of this study was to investigate the uniqueness of MPS IVA patients in Latin America in comparison with other ethnic populations and usefulness of KS concentration as a biomarker. We report 12 different mutations in this panel of patients including nine novel mutations; characterization of p.R386C, the most common mutation distributed in the pan-ethnic populations; and correlation among genotype, phenotype, and KS.

\section{Materials and methods}

Patients

MPS IVA patients were evaluated clinically. Clinical diagnosis was confirmed by fibroblasts having very low levels of GALNS activity (under 1\% of enzyme activity of normal control). Patient clinical data are reported in Table 1. Seven, seven, four, and two DNA samples were collected from Argentina, Brazil, Colombia, and Chile, respectively. Two sibling cases (patients 10 and 11 and patients 14 and 15) were included. Patients 18 and 20 were the results of consanguinity. Other patients were neither related to each other nor born from a consanguineous marriage. A written informed consent was obtained for each patient at the entry of the study at the respective center. The study protocol was approved by the IRB at Saint Louis University Hospital.

Mutation analysis of GALNS gene and KS assay

DNAs of MPS IVA patients were isolated from the peripheral blood or fibroblasts. To investigate the GALNS gene, genomic PCR was performed. The GALNS gene exons and exon-intron boundaries were amplified by using primers and PCR conditions as previously described (Ogawa et al. 1995). In the amplification of exon 1, 5\% dimethylsulfoxide (DMSO) was added. The PCR products were directly sequenced using fluorescent-labeled dideoxynucleotides. Haplotype analyses were done by using six restriction enzyme sites as previously described (Iwata et al. 1995; Tomatsu et al. 1998). Plasma and urine KS concentrations were measured using the ELISA-sandwich method, and the reagents were obtained from Seikagaku (Tokyo, Japan) (Tomatsu et al. 2004).
Construction of mutant expression vector and transient transfection into enzyme-deficient fibroblasts

To examine the relationship between the mutation and the GALNS catalytic activity, the site-directed mutagenesis was done by constructing the mutant expression vectors of $\mathrm{p} . \mathrm{R} 380 \mathrm{~S}$ and $\mathrm{p} . \mathrm{R} 386 \mathrm{C}$ mutations according to the supplier's recommendations (Stratagene, La Jolla, CA, USA) using the wild GALNS cDNA subcloned into pBS Bluescript.

The sequences of the mutagenic primers used were:

p.R380S-S: 5'-CGGCTGATGGACAGTCCTATCTTCTATTA- $3^{\prime}$

p.R380S-R: 5'-TAATAGAAGATAGGACTGTCCATCAGCCG-3'

p.R386C-S: 5'-CCTATCTTCTATTACTGTGGCGACACGCTG-3'

p.R386C-R: 5'-CAGCGTGTCGCCACAGTAATAGAAGATAGG-3'

The presence of the mutation in the construct was confirmed by sequencing analysis. The mutant cDNAs were cloned into the eukaryotic expression vector pCXN derived from pCAGGS (Miyazaki et al. 1991). Four micrograms of p.R380S, p.R386C, and wild type GALNS cDNA expression vectors were transiently transfected into GALNS-deficient fibroblasts by the lipofectamine transfection method (GIBCO BRL) according to the manufacturer's instructions. The fibroblasts were collected $24 \mathrm{~h}$ after transfection. The pellet of fibroblasts was resuspended into $200 \mu \mathrm{l}$ of $10 \mathrm{mM}$ Tris $-\mathrm{HCl}(\mathrm{pH}$ 7.0). Samples were sonicated and centrifuged for $10 \mathrm{~min}$ at $4^{\circ} \mathrm{C}$. Protein concentration and GALNS activities were measured in the cell supernatants as previously described (Montano et al. 2003).

\section{Results}

Clinical diagnoses and mutation analyses of MPS IVA patients

We analyzed the GALNS genes of 20 MPS IVA patients from Latin America using genomic PCR and direct sequencing method. Clinical characteristics and identified genotypes are summarized in Table 1. On physical examination, all patients were found to have growth retardation and bone dysplasia, and some were found to have heart valvular disease and deafness. The final height of the patients who had arrested growth was between $95 \mathrm{~cm}(-6.4 \mathrm{SD})$ and $141 \mathrm{~cm}(-5.3 \mathrm{SD})$. Based on physical examination, three patients had an attenuated form, and the other 12 had a severe form.

As a result of DNA sequence analysis, 11 different missense mutations and one insertion were identified. Thirty-six of the 40 mutant alleles were defined, and four alleles remained to be identified. All identified mutations accounted for $90.0 \%(36 / 40)$ of the mutant alleles. Nine of the 12 mutations [c.224C $>$ G (p.A75G), c.236G $>A$ 


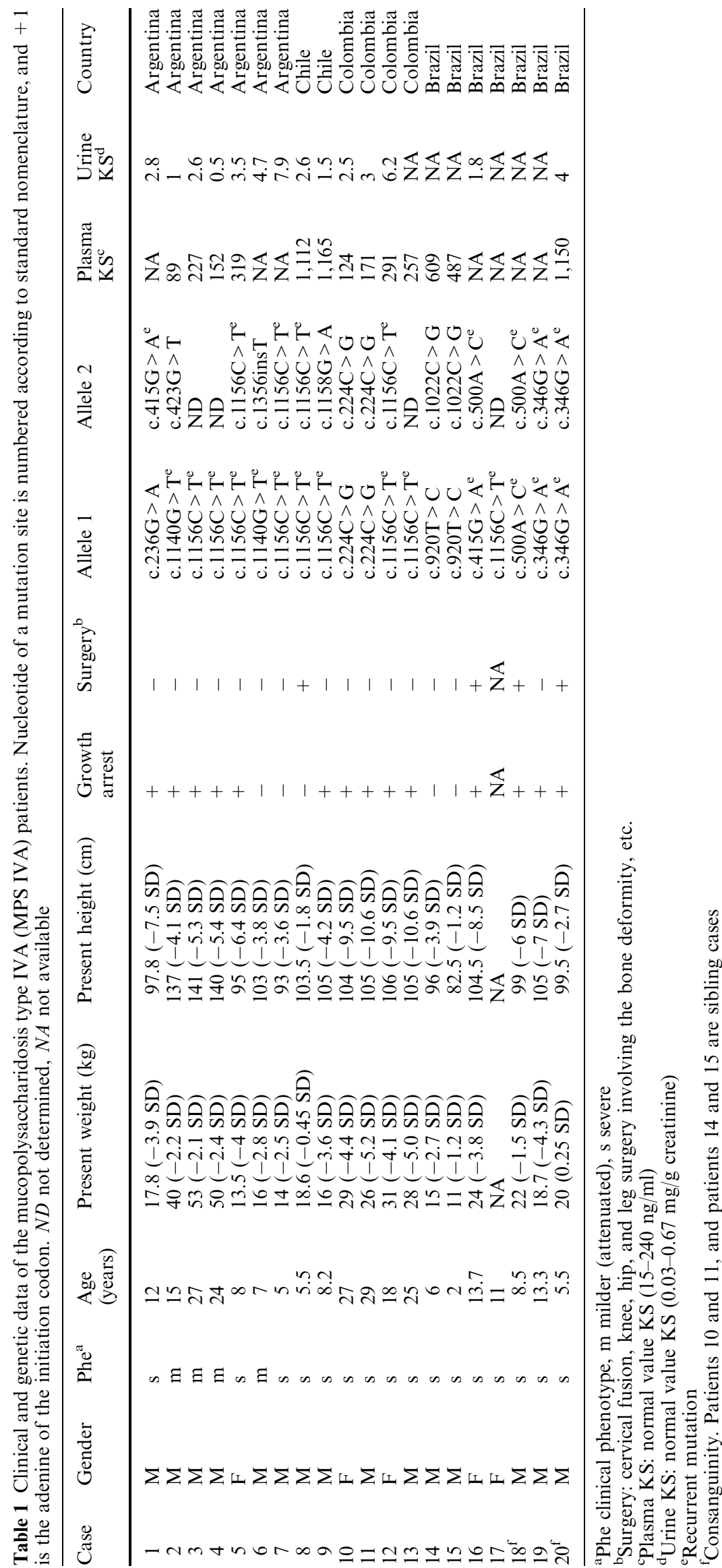


(p.C79Y), c.346G $>$ A (p.G116S), c.423G $>$ T (p.W141C), c.500A > C (p.N164T), c.920T > C (p.L307P), c. $1022 \mathrm{C}>\mathrm{G} \quad$ (p.S341R), c.1158G $>$ A (p.R386H), and c.1356insT] are described here for the first time. Three c.415G > A (p.G139S), c.1140G > T (p.R380S), and c.1156C $>$ T (p.R386C) were previously reported (Tomatsu et al. 1997, 1998). None were found in over 100 normal chromosomes of Latin American ancestry. In this population investigated, six recurrent mutations were found (p.A75G, 5.0\%; p.G116S, 10.0\%; p.G139S, $5.0 \%$; p.N164T, 7.5\%; p.R380S， 5.0\%; p.R386C, $32.5 \%$ ). Four out of 11 point mutations (p.G116S, p.G139S, p.R386H, p.R386C) resulted from transitions at $\mathrm{CpG}$ dinucleotide sites. These $\mathrm{CpG}$ transitional mutations accounted for 20 out of 40 unrelated mutant alleles $(50.0 \%)$. Haplotype analyses for the most common mutation, p.R386C, revealed the same haplotype (abhcDe) in all four homozygous patients from three different countries (patients 5, 7, 8, and 12).

\section{Expression analysis}

The p.R380S and p.R386C mutant proteins were expressed in the deficient fibroblasts, and GALNS activity in the cell supernatants was determined. Cell supernatants of the mutant p.R386C shown no detectable activity $(<1 \mathrm{nmol} / \mathrm{h} / \mathrm{mg}$ protein, $n=5)$. The mutant p.R380S had residual activity of $5.4 \%$ of the wild type GALNS activity $(5.0 \pm 0.4 \mathrm{nmol} / \mathrm{h} / \mathrm{mg}$ protein versus $93.0 \pm 4 \mathrm{nmol} / \mathrm{h} / \mathrm{mg}$ protein, $n=5)$.

Relation among genotype, phenotype, and $\mathrm{KS}$ concentration

Nine patients (patients 5, 7, 8,10-12, 18-20) were homozygous for the respective mutation. One mutation (p.R380S) was associated with a milder phenotype, while 11 other mutations were associated with a severe clinical phenotype of each patient, homozygosity of the mutation, and the expression studies for p.G139S, p.R380S, and p.R386C (Tomatsu et al. 1997; Sukegawa et al. 2000; the current study).

Plasma KS concentrations in MPS IVA patients examined were between $89 \mathrm{ng} / \mathrm{ml}$ and $1,165 \mathrm{ng} / \mathrm{ml}$. Values in patients under 15 years were higher than those in age-matched controls (mean $807 \mathrm{ng} / \mathrm{ml}$ versus $195 \mathrm{ng} /$ $\mathrm{ml}$ for MPS IVA patients and controls, respectively), while those in patients over 15 years were nearly normal (mean $187 \mathrm{ng} / \mathrm{ml}$ versus $166 \mathrm{ng} / \mathrm{ml}$ for MPS IVA patients and controls, respectively) (Table 1). Urine KS concentrations in MPS IVA patients $(0.5-7.9 \mathrm{mg} / \mathrm{g}$ creatinine) were also significantly higher than those of agematched controls (mean in all ages, $0.21 \mathrm{mg} / \mathrm{g}$ creatinine) (Tomatsu et al. 2004). Plasma KS concentrations in severe MPS IVA patients $(n=9)$ were 3.5 times higher than those in attenuated patients $(n=4)$ (mean, $569 \mathrm{ng} /$ $\mathrm{ml}$ versus $156 \mathrm{ng} / \mathrm{ml}$ ). Urine $\mathrm{KS}$ in severe MPS IVA patients was also higher than that in attenuated patients (mean, $3.6 \mathrm{mg} / \mathrm{g}$ vs $2.2 \mathrm{mg} / \mathrm{g}$ creatinine). Four severe patients homozygous with the p.R386C mutation had an elevation of plasma KS (mean $574 \mathrm{ng} / \mathrm{ml}$ ) and urine $\mathrm{KS}$ (mean $5.0 \mathrm{mg} / \mathrm{g}$ creatinine).

\section{Discussion}

We characterized the mutations of 20 Latin American patients with MPS IVA and have shown the allelic heterogeneity reflecting the wide spectrum of clinical phenotypes of MPS IVA. Sequence alterations in the GALNS gene resulted in nine novel and three previously reported mutations. The p.R386C mutation was found as a homozygous state in four patients from Argentina, Colombia, and Chile, and a heterozygous state in four patients from all four countries. The p.R386C (CGT to TGT) mutation at the $\mathrm{CpG}$ dinucleotide, nonconservative, amino acid change generates a large structural alteration of GALNS protein (Sukegawa et al. 2000) leading to a severe form of MPS IVA. This allele frequency was found to be highest among unrelated mutant alleles in this study. The finding of the same haplotype on the p.R386C mutation suggests that it was derived from the same founder. The p.R386C mutation was also found in other ethnic groups such as Japanese, British, Italian, Turkish, German, Polish, and Mexican, accounting for $2-8 \%$, although the haplotypes on the mutant alleles were different from those in Latin American patients. The p.G139S (GGC to AGC), another transitional mutation at the $\mathrm{CpG}$ dinucleotide, was also found in several ethnic groups (Argentina, Brazil, UK, and USA).

The p.G116S (GGC to AGC), a transitional mutation at $\mathrm{CpG}$ dinucleotide, was identified in two unrelated Brazilian patients in a homozygous state. The p.A75G (G $C$ C to $\mathrm{G} G \mathrm{C}$ ) and p.N164T (AAC to ACC) mutations at the nonCpG dinucleotide were found in only Colombian and Brazilian patients, respectively. The p.R380S (AGG to AGT) mutation found in two Argentine patients was also identified in an Italian MPS IVA patient with a milder form (data not shown). Further haplotype studies for each patient family are needed to clarify whether each frequent mutation in this study is truly recurrent or identical by descent.

Missense mutations, except p.R380S, are shown to be associated with a severe phenotype. An in vitro expression study supported p.R380S being a milder phenotype. We have not analyzed the effect of the newly found mutations with an expression study except for p.R380S; instead, we have compared the places where missense mutations occurred with the conserved domains among human lysosomal sulfatases, since the conserved domains among sulfatases are essential for the common sulfatase activity (Dierks et al. 2003). The Cysteine 79 residue is conserved among all eukaryotic sulfatases. This cysteine is posttranslationally converted to formylglycine in the endoplasmic reticulum by the sulfatase 
modifying factor 1 (Dierks et al. 2003; Cosma et al. 2003). Formylglycine is the catalytic residue in the active site of eukaryotic sulfatases. The p.C79Y mutation detected in patient 1 was the first mutation ever found at the catalytic site, leading to a severely affected MPS IVA phenotype. Five mutations (p.A75G, p.G116S, p.G139S, p.W141C, and p.S341R) defining a severe phenotype also occurred on the conserved amino acid residues. The other five missense mutations (p.N164T, p.L307P, p.R380S, p.R386H, and p.R386C) were located on the nonconserved amino acid residues with nonconservative amino acid changes. A large (nonconservative) amino acid change also provides deformation of the secondary and/or tertiary structure of the GALNS protein, leading to the clinical phenotype. None of the missense mutations here were a nonconserved amino acid residue with a conservative amino acid change. These factors partly explain why these mutations caused MPS IVA, mainly in a severe form.

Urine and plasma KS concentrations were measured in some patients. The patients investigated had an elevation of urine and plasma KS concentrations compared with those of the age-matched control (especially under 15 years old). Severe patients had a higher elevation of $\mathrm{KS}$ compared with the attenuated patients. These findings suggest that KS concentration in urine and blood is a potential biomarker for clinical severity, albeit modified with age. Further studies including a larger number of cases of the same age and genotype are needed to clarify the correlation between a certain genotype and the extent of elevation of KS.

In conclusion, this study provides further information on allelic heterogeneity of MPS IVA in each ethnic population as well as the significance of recurrent mutations at the $\mathrm{CpG}$ dinucleotide site. Accumulation of novel mutations with clinical description and KS concentration in urine and blood will lead us to predict the prognosis of the patient more precisely.

Acknowledgements This work was supported by grants from the Austrian Research Society for Mucopolysaccharidoses and Related Diseases, German MPS, Italian MPS, and International Morquio Organization (Carol Ann Foundation).

\section{References}

Bunge S, Kleijer WJ, Tylki-Szymanska A, Steglich C, Beck M, Tomatsu S, Fukuda S, Poortuis BJHM, Czartoryska B, Orii T, Gal A (1997) Identification of 31 novel mutations in the $N$ acetylgalactosamine-6-sulfate sulfatase gene reveals excessive allelic heterogeneity among patients with Morquio A syndrome. Hum Mutat 10:223-232

Cosma MP, Pepe S, Annunziata I, Newbold RF, Grompe M, Parenti G, Ballabio A (2003) The multiple sulfatase deficiency gene encodes an essential and limiting factor for the activity of sulfatases. Cell 113:445-456

Dierks T, Schmidt B, Borissenko LV, Peng J, Preusser A, Mariappan M, von Figura K (2003) Multiple sulfatase deficiency is caused by mutations in the gene encoding the human C(alpha)formylglycine generating enzyme. Cell 113:435-444
Fukuda S, Tomatsu S, Masue M, Sukegawa K, Iwata H, Ogawa T, Nakashima Y, Hori T, Yamagishi A, Hanyu Y, Morooka K, Kiman T, Hashimoto T, Orii T (1992) Mucopolysaccharidosis type IVA $N$-acetylgalactosamine-6-sulfate sulfatase exonic point mutation in classical Morquio and mild cases. J Clin Invest 90:1049-1053

Iwata $\mathrm{H}$, Tomatsu S, Fukuda S, Uchiyama A, Rezvi GM, Ogawa T, Hori T, Nakashima Y, Yamagishi A, Sukegawa K (1995) Mucopolysaccharidosis IVA: polymorphic haplotypes and informative RFLPs in the Japanese population. Hum Genet 95:257-264

Montano AM, Kaitila I, Sukegawa K, Tomatsu S, Kato Z, Nakamura H, Fukuda S, Orii T, Kondo N (2003) Mucopolysaccharidosis IVA: characterization of a common mutation found in Finnish patients with attenuated phenotype. Hum Genet 113:162-169

Nakashima Y, Tomatsu S, Hori T, Fukuda S, Sukegawa K, Kondo N, Suzuki Y, Shimozawa N, Orii T (1994) Mucopolysaccharidosis IVA: molecular cloning of the human $N$-acetylgalactosamine-6-sulfate sulfatase gene (GALNS) and analysis of the 5'flanking region. Genomics 20:99-104

Ogawa T, Tomatsu S, Fukuda S, Yamagishi A, Rezvi GMM, Sukegawa K, Kondo N, Suzuki Y, Shimozawa N, Orii T (1995) Mucopolysaccharidosis IVA: screening and identification of mutations of the $\mathrm{N}$-acetylgalactosamine-6-sulfate sulfatase gene. Hum Mol Genet 4:341-349

Sukegawa K, Nakamura H, Kato Z, Tomatsu S, Montano AM, Fukao T, Toietta G, Tortora P, Orii T, Kondo N (2000) Biochemical and structural analysis of missense mutations in $\mathrm{N}$ acetylgalactosamine-6-sulfate sulfatase causing mucopolysaccharidosis IVA phenotypes. Hum Mol Genet 9:1283-1290

Tomatsu S, Fukuda S, Masue M, Sukegawa K, Fukao T, Yamagishi A, Hori T, Iwata H, Ogawa T, Nakashima Y, Hanyu Y, Hashimoto T, Titani K, Oyama R, Suzuki M, Yagi K, Hayashi Y, Orii T (1991) Morquio disease: isolation, characterization and expression of full length cDNA for human $\mathrm{N}$ acetylgalactosamine-6-sulfate sulfatase. Biochem Biophys Res Commun 181:677-683

Tomatsu S, Fukuda S, Cooper A, Wraith JE, Rezvi GMM, Yamagishi A, Yamada N, Kato Z, Isogai K, Sukegawa K, Kondo N, Suzuki Y, Shimozawa N, Orii T (1995) Mucopolysaccharidosis IVA: identification of a common missense mutation $\mathrm{I} 113 \mathrm{~F}$ in the $N$-acetylgalactosamine-6-sulfate sulfatase gene. Am J Hum Genet 57:556-563

Tomatsu S, Fukuda S, Cooper A, Wraith JE, Ferreira P, Di Natale P, Tortora P, Fujimoto A, Kato Z, Yamada N, Isogai K, Yamagishi A, Sukegawa K, Suzuki Y, Shimozawa N, Kondo N, Sly WS, Orii T (1997) Fourteen novel mucopolysaccharidosis IVA producing mutations in GALNS gene. Hum Mutat $10: 368-375$

Tomatsu S, Fukuda S, Cooper A, Wraith JE, Yamagishi A, Kato Z, Yamada N, Isogai K, Sukegawa K, Suzuki Y, Shimozawa $\mathrm{N}$, Kondo N, Orii T (1998) Fifteen polymorphisms in the $\mathrm{N}$-acetylgalactosamine-6-sulfate sulfatase (GALNS) gene: diagnostic implications in Morquio disease. Hum Mutat Suppl 1:S42-S46

Tomatsu S, Okamura K, Taketani T, Orii KO, Nishioka T, Gutierrez MA, Velez-Castrillon S, Fachel AA, Grubb JH, Cooper A, Thornley M, Wraith E, Barrera LA, Giugliani R, Schwartz IV, Frenking GS, Beck M, Kircher SG, Paschke E, Yamaguchi S, Ullrich K, Isogai K, Suzuki Y, Orii T, Kondo N, Creer M, Noguchi A (2004) Development and testing of new screening method for keratan sulfate in mucopolysaccharidosis IVA. Pediatr Res 55:592-597

Yamada N, Fukuda S, Tomatsu S, Muller V, Hopwood JJ, Nelson J, Kato Z, Yamagishi A, Sukegawa K, Kondo N, Orii T (1998) Molecular heterogeneity in mucopolysaccharidosis IVA in Australia and Northern Ireland: nine novel mutations including T312S, a common allele that confers a mild phenotype. Hum Mutat 11:202-208 